\title{
ON THE PRIME IDEALS OF SMALLEST NORM IN AN IDEAL CLASS mod $f$ OF AN ALGEBRAIC NUMBER FIELD
}

\author{
BY G. J. RIEGER \\ Communicated by I. J. Schoenberg, February 3, 1961
}

In 1947, Linnik [3] proved the following theorem:

TheOREM (OF LinNiK). There exists an absolute constant $c$ such that in every prime residue class mod $k$ there is a prime number $p$ with $p<k^{c}$.

A simplified proof of this theorem was given by Rodosskii [7] whose proof (similar to Linnik's) rests basically on (A) functiontheoretic lemmas, (B) theorems on $L$-functions, (C) estimates of character sums, and (D) a sieve method. The theorems (B) can be classified and characterized as follows: 5.4],

(B1) order of magnitude of the $L$-functions [5, Chapter 4, Satz $6.9]$,

(B2) existence of at most one exceptional zero [5, Chapter 4, Satz 8.1],

(B3) Siegel's theorem on the exceptional zero [5, Chapter 4, Satz 1.1],

(B4) functional equation of the $L$-functions [5, Chapter 7 , Satz

(B5) number of zeros in vertical strips [5, Chapter 7, Satz 3.3],

(B6) explicit formulae [5, Chapter 7, Satz 4.1, Satz 6.1].

Recently, I have been able to prove the following generalization of Linnik's theorem which I had conjectured elsewhere [6, p. 168]:

THEOREM 1. For every algebraic number field $K$ there exists a constant $c(K)$, depending on $K$ only, such that in every ideal class mod $f$ (in the narrowest sense) there is a prime ideal $p$ with $N p<N \mathfrak{f}^{(K)}$.

The skeleton of the proof of Theorem 1 can be taken from Rodosskii's proof; the lemmas (A) are the same; the generalized theorems (B1) resp. (B3) resp. (B4) resp. (B5) resp. (C) resp. (D) can be found in [1] and [4] resp. [4] resp. [1] resp. [1] resp. [2] resp. [6]; the remaining theorems (B2) and (B6) can easily be generalized. The details of the proof of Theorem 1 are then essentially the same as in [7]. This completes the outline of the proof of Theorem 1. 
To the related question of the "smallest" prime numbers in a residue class mod $f$ we hope to come back soon.

\section{BIBLIOGRAPHY}

1. E. Landau, Ueber Ideale und Primideale in Idealklassen, Math. Z. vol. 2 (1918) pp. 52-154.

2. - Verallgemeinerung eines Pólyaschen Satzes auf algebraische Zahlkoerper, Nachr. Akad. Wiss. Goettingen (1918) pp. 478-488.

3. J. V. Linnik, Ueber die kleinste Primzahl in einer arithmetischen Progression, Mat. Sb. vol. 15 (1947) pp. 139-368.

4. T. Mitsui, Generalized prime number theorem, Jap. J. Math. vol. 26 (1956) pp. $1-42$.

5. K. Prachar, Primzahlverteilung, Berlin, Springer-Verlag, 1957.

6. G. J. Rieger, Verallgemeinerung der Siebmethode von A. Selberg auf algebraische Zahlkoerper. II, J. Reine Angew. Math. vol. 201 (1959) pp. 157-171.

7. K. A. Rodosskii, Ueber die kleinste Primzahl in einer arithmetischen Progression, Mat. Sb. vol. 34 (1954) pp. 331-356.

Purdue University 